\title{
NANOESPECTROSCOPIA DE ABSORÇÃO DE RADIAÇÃO SÍNCROTRON NO INFRAVERMELHO PARA NANOESPECIAÇÃO DE PRODUTOS DE CORROSÃO METÁLICA
}

\author{
Thiago S. Puglieria, ${ }^{a, *,}$,, Raul de O. Freitas ${ }^{\mathrm{b}}$, Francisco C. B. Maia ${ }^{\mathrm{b}}$ e Dalva L. A. de Faria ${ }^{\mathrm{a}}$ \\ anstituto de Química, Universidade de São Paulo, 05508-000 São Paulo - SP, Brasil \\ ${ }^{b}$ Laboratório Nacional de Luz Síncrotron, Centro Nacional de Pesquisa em Energia e Materiais, 13083-970 Campinas - SP, Brasil \\ 'Departamento de Museologia, Conservação e Restauro, Universidade Federal de Pelotas, 96010-280 Pelotas - RS, Brasil
}

Recebido em 17/05/2019; aceito em 10/09/2019; publicado na web em 21/10/2019

\begin{abstract}
SYNCHROTRON INFRARED NANO-SPECTROSCOPY FOR NANO-SPECIATION OF METAL CORROSION PRODUCTS. The understanding of chemical and physical behavior of materials, especially metals and their alloys, is of utmost importance for industrial and technological applications, development of new materials and preservation of cultural heritage. The use of complementary analytical techniques has allowed a better understanding of corrosion mechanisms, and results obtained by Raman micro-spectroscopy, for example, have been complemented by FTIR and XRD. When focusing on the development of new materials and proposition of preservation strategies, the understanding of corrosion processes and of their very initial steps (nanometric scale) is fundamental. SERS reaches such lateral resolution if TERS is used, however, it cannot be universally applied and needs to be complemented by other techniques. In this work it is presented the potentials of a recent installed technique at the Brazilian Synchrotron Light Laboratory (LNLS), which can be used for nano-speciation of corrosion products, complementing investigation carried out by, for example, Raman micro-spectroscopy and TERS. It is Synchrotron Infrared Nano-Spectroscopy (SINS), which allows the investigation of both chemical nature and physical distribution of corrosion products. The infrastructure presented at LNLS is one of the few available in the world. As a model system, thin films of $\mathrm{Pb}$ exposed to formic acid were used.
\end{abstract}

Keywords: SINS; nano-FTIR; Pb corrosion; lead formate.

\section{INTRODUÇÃO}

O entendimento do comportamento químico e físico dos materiais, especialmente metais e suas ligas, desperta grande interesse devido às aplicações industriais e tecnológicas, desenvolvimento de novos materiais e preservação do patrimônio cultural, dentre outros exemplos. $\mathrm{O}$ mesmo se aplica à corrosão metálica, que usualmente envolve um grande número de reações químicas: neste caso, a especiação química dos produtos inicialmente formados e sua distribuição espacial são fundamentais para a melhor compreensão dos mecanismos envolvidos na degradação e, quando for o caso, evitá-los desde os seus estágios iniciais.

Técnicas como espectroscopia de fotoelétrons excitados por raios X (XPS, X-Ray Photoelectron Spectroscopy), espectroscopia de elétrons Auger (Auger Electron Spectroscopy), microscopia de varredura por tunelamento (STM, Scanning Tunneling Microscopy), microscopia de força atômica (AFM, Atomic Force Microscopy) e métodos eletroquímicos têm sido empregados em estudos de corrosão. ${ }^{1}$ Além dessas, técnicas espectroscópicas vibracionais, como micro-espectroscopia Raman e espectroscopia de absorção no infravermelho por transformada de Fourier (FTIR), também vêm sendo usadas. ${ }^{2}$

Baixo custo, elevada relação sinal-ruído (SNR, Signal-to-Noise Ratio), preservação de amostras, possibilidade de estudos da distribuição química em escala micrométrica, possibilidade de estudos in situ, ausência da necessidade de preparo de amostras e operação intuitiva dos equipamentos mais recentes são características que fazem das técnicas vibracionais ferramentas acessíveis e interessantes à comunidade acadêmica. Especificamente no contexto da preservação do patrimônio cultural, por exemplo, micro-espectroscopia Raman tem sido usada para a investigação de estágios iniciais de processos de corrosão de prata em atmosferas contendo compostos de enxofre, ${ }^{3}$

*e-mail: tspuglieri@ufpel.edu.br investigação de produtos e mecanismos de corrosão de objetos metálicos compostos por ferro ${ }^{4,5}$ e investigação de processos de corrosão de chumbo por formaldeído em ambientes museológicos internos. ${ }^{6,7}$

Em muitos casos há a necessidade de se investigar os processos de corrosão em escala nanométrica, seja pelo interesse em investigações isoladas de nanomateriais/nanoestruturas, seja pelo interesse em investigações de estágios muito iniciais do processo de corrosão. A micro-espectroscopia Raman apresenta uma limitação por mal alcançar resoluções sub-micrométricas devido ao limite intrínseco de difração óptica (limite de Abbe); essa limitação é mais acentuada ainda na micro-espectroscopia FTIR ( $\mu$-FTIR), por se utilizar maiores comprimentos de onda. Como alternativa para o Raman tem-se o uso de TERS (Tip Enhanced Raman Spectroscopy), ${ }^{2}$ contudo, assim como a micro-espectroscopia Raman, apresenta a necessidade de complementaridade, que neste caso poderia ser por nano-FTIR, foco deste artigo.

A complementaridade entre espectroscopia Raman e FTIR se dá por essas serem técnicas de espectroscopia vibracional que se baseiam em diferentes efeitos físicos. A espectroscopia Raman se baseia no espalhamento inelástico de radiação eletromagnética (geralmente visível, infravermelho próximo ou ultravioleta) e a FTIR na absorção de radiação eletromagnética no infravermelho (IR). Com isso, suas regras de seleção se diferenciam e, como consequência, bandas associadas a alguns modos vibracionais aparecerão com maiores intensidades numa ou noutra técnica. Em alguns casos pode ocorrer uma exclusão mútua, onde bandas que são observadas com uma técnica não as são com a outra; maiores informações podem ser obtidas em literatura específica. ${ }^{8}$ Além disso, quando TERS e nanoespectroscopia no infravermelho são comparados, essa última é menos afetada quando consideradas modificações na estrutura da ponta. ${ }^{9}$

FTIR e $\mu$-FTIR convencionais utilizam de fontes de campo distante para a obtenção de espectros, sendo que para se alcançar resoluções nanométricas é necessário o uso de fontes de campo próximo. 
s-SNOM (scattering Scanning Near-Field Optical Microscopy) ${ }^{10,11}$ é uma técnica que combina AFM e $\mu$-FTIR para alcançar resolução óptica em escala nanométrica. No s-SNOM, radiação eletromagnética é focalizada em uma ponta metálica de AFM que é polarizada e assim, via efeito antena, produz um confinamento de luz em regime de campo próximo. Dado o caráter evanescente deste confinamento, o volume confinado depende apenas do fator de forma da ponta e não mais do comprimento de onda da radiação incidente. Dado que as pontas de AFM possuem raios típicos de poucas dezenas de nanômetros, a resolução espacial da s-SNOM, mesmo na faixa de infravermelho médio, é de $\sim 25 \mathrm{~nm} .{ }^{12}$ Esta resolução pode ser eventualmente aprimorada com o desenvolvimento de pontas de AFM metálicas com raios reduzidos ou com geometrias especiais. Como consequência, os comprimentos de onda tipicamente usados no FTIR podem ser empregados para uma sondagem química com resolução lateral nanométrica.

Separação de fase de polímero em nanoescala, ${ }^{13,14}$ biominerais nano-estruturados ${ }^{15}$ e materiais bidimensionais ${ }^{16,17}$ são exemplos de campos que se beneficiam das análises de s-SNOM. Recentemente, a s-SNOM passou a ter sensibilidade química sem precedentes quando combinada com radiação sincrotron no infravermelho. Nesta configuração, denominada Synchrotron Infrared Nano Spectroscopy (SINS), esta modalidade experimental permite a análise química de materiais de forma contínua ao longo de toda a região de fingerprinting do IR com resolução espacial nanométrica, permitindo o acesso à nano química de uma variedade de materiais orgânicos e inorgânicos. ${ }^{18-22}$ Isto se dá pelo excepcional brilho das fontes de IR síncrotron, podendo ser até 1000 vezes mais brilhantes que as clássicas fontes térmicas utilizadas em FTIR ao longo de toda a faixa de IR. ${ }^{23}$

Por essas razões, SINS apresenta um considerável potencial para a investigação dos primeiros estágios de corrosão metálica e para especiação dos produtos formados, complementando análises de micro-espectroscopia Raman e permitindo identificação química ao mesmo tempo que permite obtenção de informações da distribuição espacial e morfologia dos produtos de corrosão.

O metal considerado neste estudo é o chumbo, metal dúctil e maleável, com extensivo uso no passado, variando de construções civis a materiais artísticos e objetos de uso cotidiano, como jarras. No contexto do patrimônio cultural foi amplamente utilizado em estátuas, vitrais e tubos de órgãos, entre outros. A preservação desses objetos tem estimulado várias investigações sobre os processos de degradação induzidos por fatores ambientais, como umidade relativa e poluição atmosférica, especialmente em microambientes internos. ${ }^{6,7,24,25}$ Quando presentes em ambientes com elevados níveis de umidade relativa, compostos orgânicos voláteis (VOCs, Volatile Organic Compounds) como ácidos acético e fórmico são particularmente danosos e têm sido apontados como os principais agentes de degradação de objetos de $\mathrm{Pb}$.

O entendimento desses processos de degradação dá suporte também à introdução de novas tecnologias e metodologias, incluindo monitoramento ambiental para a preservação do patrimônio. ${ }^{26}$ Entre essas tecnologias estão os dispositivos de alerta precoce, que são capazes de detectar ambientes agressivos muito antes de se observar a olho nu um processo de degradação no bem, ou seja, nos estágios iniciais de degradação. Um exemplo desses sistemas é aquele baseado em QCMs (microbalanças a cristal de quartzo, ou quartz crystal microbalances) cobertas com nanocamadas de $\mathrm{Pb}$ : quando o filme metálico é quimicamente afetado pelo ambiente, sua massa varia, alterando a frequência de oscilação do cristal. No entanto, nenhuma informação sobre os compostos químicos formados nem sobre os mecanismos de reação podem ser obtidos das respostas isoladas de frequência das QCMs. A identificação em escala nanométrica de produtos de corrosão formados quando esses filmes finos metálicos são expostos a ambientes quimicamente agressivos é o primeiro passo para o entendimento dos mecanismos de degradação e proposição de estratégias de conservação preventiva ou mitigação. SINS, neste caso, é uma técnica capaz de produzir informações físicas e químicas desses estágios muito iniciais dos processos de corrosão, auxiliando na preservação do patrimônio.

Neste trabalho, com o objetivo de demonstrar o potencial de SINS como técnica complementar à micro-espectroscopia Raman na investigação de corrosão metálica, para nanoespeciação dos produtos de corrosão, utilizou-se de $\mathrm{Pb}$ corroído por ácido fórmico como sistema modelo. Na investigação também se empregou AFM, IR s-SNOM, micro-espectroscopia Raman e FTIR.

Destaca-se que, embora o potencial de SINS já esteja sendo demonstrado em diversos campos do conhecimento,,${ }^{9,27-30}$ tal técnica ainda não está sendo usada de forma sistemática na investigação de bens culturais, ao contrário de $\mu$-FTIR com radiação sincrotron. ${ }^{31,32}$ Atualmente, além do Laboratório Nacional de Luz Síncrotron (LNLS), apenas 2 laboratórios no mundo realizam o experimento SINS de forma aberta à comunidade. ${ }^{19,33,34}$ Este experimento tem auxiliado investigações em múltiplas disciplinas na comunidade brasileira de materiais e em breve dará mais um salto de sensibilidade (até 10 vezes maior) com sua transferência para o novo acelerador brasileiro, o Sirius.

\section{EXPERIMENTAL}

\section{Superfícies de $\mathbf{P b}$}

Superfícies de $\mathrm{Pb}$ com espessuras nanométricas (da ordem de $20 \mathrm{~nm}$ ) foram preparadas por sputtering de $\mathrm{Pb}$ (Goodfellow Cambridge Ltd., 99.99+\%) sobre lâminas de Si (5 x 5 mm, Ted Pella, Inc.) usando um equipamento Scancoat Six (Edwards). A pressão do plasma $\left(\mathrm{Ar}^{+}\right)$foi mantida constante e um pouco acima de $0,2 \mathrm{mbar}$, enquanto o potencial foi mantido em $c a$. $0,75 \mathrm{kV}$. Diferentes tempos de exposição (de um a cinco minutos) foram usados para produzir filmes com espessuras variadas.

Os filmes de $\mathrm{Pb}$ foram expostos por 40 minutos a atmosferas criadas com $5 \mathrm{~mL}$ de ácido fórmico (Synth, grau analítico) e 54\% de umidade relativa, utilizando-se de $c a .50 \mathrm{~mL}$ de solução aquosa saturada de $\mathrm{Mg}\left(\mathrm{NO}_{3}\right)_{2} \cdot 6 \mathrm{H}_{2} \mathrm{O}$ (Riedel-de Haën, 99.5+\%).

As nanoestruturas corroídas foram caracterizadas por AFM, s-SNOM, SINS e micro-espectroscopia Raman.

\section{Síntese e caracterização de formiatos padrões de $\mathbf{P b}$}

Diferentes formiatos de $\mathrm{Pb}$ (aqui identificados como formiatos 1 a 4) foram sintetizados hidrotermicamente usando cada um dos conjuntos de reagentes mostrados na Tabela $1{ }^{35}$ As sínteses hidrotérmicas foram realizadas mantendo-se os reagentes em uma autoclave envolta por Teflon ${ }^{\circledast}$ a $110{ }^{\circ} \mathrm{C}$ por aproximadamente $12 \mathrm{~h}$. Antes do aquecimento as misturas foram mantidas em banho de ultrassom por $c a$. 20 minutos. Após resfriamento, até temperatura ambiente, as autoclaves foram abertas e os cristais secos ao ar, sendo separados manualmente para caracterização. Os formiatos de $\mathrm{Pb}$ foram caracterizados por micro-espectroscopia Raman e FTIR-ATR.

\section{Especificações instrumentais}

Um microscópio Raman da Renishaw (System 3000), composto por um monocromador de $25 \mathrm{~cm}$, equipado com uma rede holográfica de 1800 linhas/mm e acoplado a um detector CCD termoeletricamente refrigerado (600 x 400 pixels, Andor) foi usado nas medidas Raman. Um microscópio Leica (DM2500M) foi usado para focalizar o feixe de laser e coletar a radiação espalhada da amostra. A excitação foi 
Tabela 1. Informações sobre a síntese dos formiatos de $\mathrm{Pb}$

\begin{tabular}{|c|c|}
\hline $\begin{array}{c}\text { Formiato } \\
\text { (PbO:HCOOH razão molar) }\end{array}$ & Reagente \\
\hline $\begin{array}{l}\text { Formiato } 1 \\
\quad(1: 2)\end{array}$ & $\begin{array}{c}0,738 \mathrm{~g}(3,30 \mathrm{mmol}) \mathrm{PbO}+ \\
0,700 \mathrm{~mL} \mathrm{HCOOH}(\mathrm{aq}) 9,44 \mathrm{~mol} \mathrm{~L}^{-1} \\
(6,60 \mathrm{mmol})+5,00 \mathrm{~mL} \text { de água } \\
\text { deionizada }\end{array}$ \\
\hline $\begin{array}{l}\text { Formiato } 2 \\
\quad(1: 1)\end{array}$ & $\begin{array}{c}0,738 \mathrm{~g}(3,30 \mathrm{mmol}) \mathrm{PbO}+ \\
0,700 \mathrm{~mL} \mathrm{HCOOH}(\mathrm{aq}) 4,72 \mathrm{~mol} \mathrm{~L}^{-1} \\
(3,30 \mathrm{mmol})+5,00 \mathrm{~mL} \text { de água } \\
\text { deionizada }\end{array}$ \\
\hline $\begin{array}{c}\text { Formiatos } 3 \text { e } 4 \\
\qquad(2: 1)\end{array}$ & $\begin{array}{c}0,738 \mathrm{~g}(3,30 \mathrm{mmol}) \mathrm{PbO}+ \\
0,700 \mathrm{~mL} \mathrm{HCOOH}(\mathrm{aq}) 2,36 \mathrm{~mol} \mathrm{~L}^{-1} \\
(1,65 \mathrm{mmol})+5,00 \mathrm{~mL} \text { de água } \\
\text { deionizada }\end{array}$ \\
\hline
\end{tabular}

obtida com laser He-Ne em 632,8 nm (Coherent).

Experimentos SINS (Figura 1a) foram realizados na linha IR1 do LNLS que usa radiação IR de borda e contínua de um dipolo de 1,67 T. O feixe é extraído e colimado por ópticas dedicadas à esta linha ${ }^{36,37}$ chegando ao microscópio s-SNOM (NeaSnom, NeaSpec $\mathrm{GmbH})$. No microscópio, um estágio AFM especial, acoplado a lentes de focalização, é responsável pela varredura da amostra que interage com os campos elétricos ultraconfinados no ápice da ponta de AFM. O sistema ponta-amostra é montado em um dos braços de um interferômetro assimétrico de Michelson, que é responsável pela sensibilidade à fase óptica do s-SNOM. Um espelho de referência cria interferogramas que, após processamento por transformada de Fourier, geram espectros de banda larga. Análises AFM e SINS foram feitas usando pontas AFM recobertas com Pt, com ressonância mecânica em ca. 250 kHz (NC-Pt ARROW, NanoWorld). Um detector de telureto de mercúrio e cádmio (MCT, Kolmar Technologies) foi usado tanto para imageamento de banda larga no IR quanto para espectros pontuais de SINS. Supressão de fundo via demodulação de harmônicas mais altas ${ }^{12}$ foi feita pelo amplificador lock-in NeaSnom, quando usada a dinâmica da ponta do AFM como referência para a detecção do sinal do detector MCT.

Como mostrado na Figura 1a, o feixe colimado de IR de banda larga é dirigido por óptica primária a um instrumento comercial s-SNOM. O instrumento s-SNOM é um AFM onde o feixe de IR incidente é focalizado em uma ponta de AFM, que age como uma antena para o campo elétrico incidente, produzindo um amplo aumento da radiação incidente no ápice da ponta. Com isso, uma fonte nanométrica de IR muito brilhante (também conhecida como fonte de campo próximo) é criada e seu tamanho é comparável ao raio da ponta e não mais depende do comprimento de onda. ${ }^{38} \mathrm{O}$ AFM é programado para trabalhar no modo de semi-contato (tapping mode), onde a ponta oscila a $250 \mathrm{kHz}$. Consequentemente, uma interação óptica de campo próximo entre a ponta e a amostra ocorre simultaneamente às medidas topográficas por AFM, gerando uma onda espalhada, harmonicamente sincronizada com a oscilação da ponta. Um detector MCT com resposta espectral de $570 \mathrm{~cm}^{-1}$ a $5000 \mathrm{~cm}^{-1}$ e eficiência máxima em $1000 \mathrm{~cm}^{-1}$, conectado a um amplificador lock-in, é usado para detecção de harmônicas mais altas de $\omega$, de modo a filtrar o sinal de campo próximo do sinal produzido de campo distante por um espalhamento geral do eixo da ponta e da amostra. Além de imagens IR de banda larga, que podem ser obtidas pelo monitoramento de sinal retro-espalhado da ponta quando varre a superfície da amostra, o microscópio é também capaz de realizar medidas espectroscópicas, desde que a ponta e a amostra estejam acoplados a um dos braços de um interferômetro assimétrico de Michelson, como ilustrado na Figura 1. Neste caso, o espelho de referência varia o passo óptico do feixe de referência que interfere com o sinal retro-espalhado da ponta-amostra após o beamsplitter. O sinal coletado como um interferograma é então processado matematicamente (transformada de Fourier) e o software do microscópio produz espectros de amplitude e fase da amostra, como mostrados nas figuras seguintes.

Para medir os espectros SINS, 20 co-adições foram consideradas em cada ponto, sendo que cada espectro foi obtido em 3,5 minutos com resolução espectral de $6 \mathrm{~cm}^{-1}$. Para suprimir contribuições da fonte, da resposta do detector e do ambiente, os espectros obtidos na varredura em linha foram normalizados por um espectro de $\mathrm{Si}$.

Espectros FTIR-ATR foram obtidos num equipamento Bruker, modelo Alpha, com óptica de $\mathrm{KBr}$ e detector de DTGS (deuterated triglycine sulfate), utilizando-se de um acessório também Bruker de única reflexão (Platinum - ATR com cristal de diamante).

Os espectros Raman e FTIR-ATR foram analisados com o pacote computacional Grams (Thermo Sci. Inc.) e/ou OriginPro 8 (OriginLab Co.). As imagens de IR de banda larga e de topografia obtidas durante medidas de s-SNOM e AFM foram analisadas com o programa Gwyddion (http://gwyddion.net).

\section{RESULTADOS E DISCUSSÃO}

Lâminas de Si foram escolhidas como substrato para filmes de $\mathrm{Pb}$ porque a técnica SINS demanda superfícies planas, por ser baseada
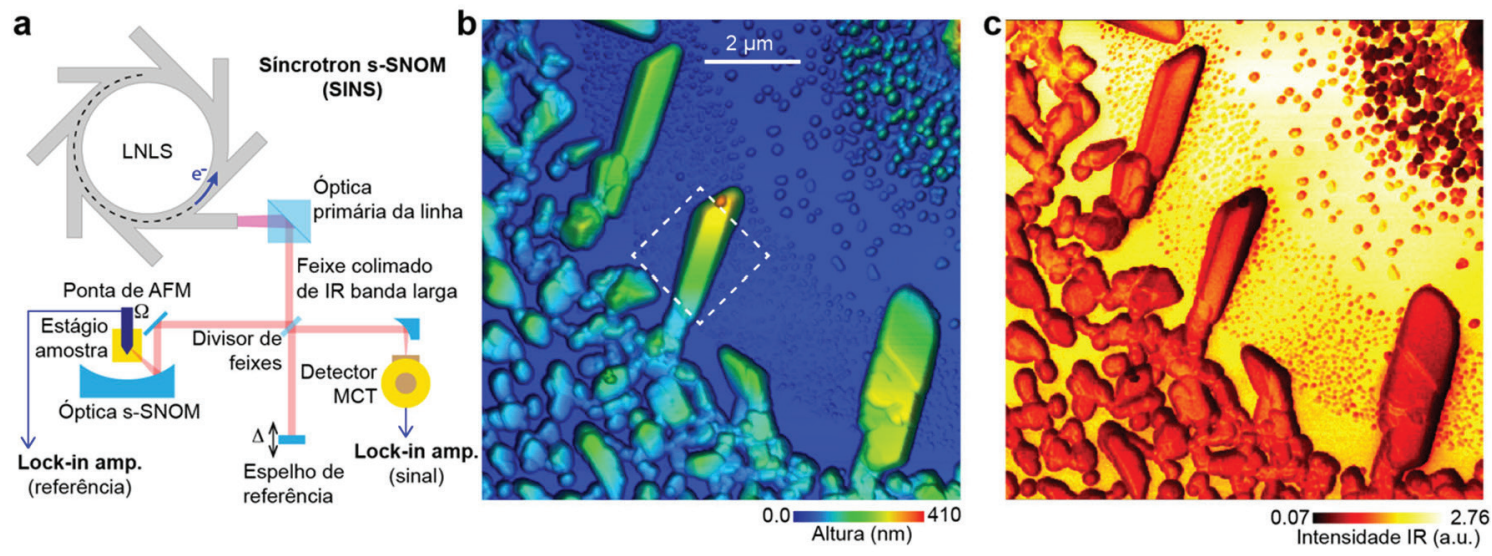

Figura 1. (a) Diagrama do experimento SINS em que espectroscopia de absorção no IR com radiação síncrotron e AFM são combinadas para análises químicas locais de materiais. (b) Imagem da topografia por AFM de um filme de Pb sobre Si após o procedimento controlado de corrosão. A morfologia da superfície mostra diferentes padrões de nanoestruturas. Um quadrado branco pontilhado indica a área analisada e mostrada na Figura 2 e na Figura 3 . (c) Mapa de IR de banda larga, medido simultaneamente com a imagem de topografia de AFM, mostrando o respectivo contraste óptico entre as diferentes estruturas após corrosão 
em um arranjo de AFM. Além disso, $\mathrm{Si}$ apresenta uma resposta espectral plana no IR, não interferindo nas medidas. Deposição por sputtering foi escolhida para a preparação da superfície metálica porque produz filmes finos nano-cristalinos e nano-partículas fracamente agregadas. ${ }^{39}$ Assim que é exposto ao ambiente, o filme fino de $\mathrm{Pb}$ é rapidamente oxidado, formando majoritariamente $\mathrm{PbO}$, o qual foi prontamente atacado na atmosfera contendo ácido fórmico e os produtos formados foram analisados por AFM e SINS.

A Figura 1b mostra a imagem AFM e a Figura 1c mostra o mapa de IR de banda larga, evidenciando os produtos de corrosão que são muito diferentes em forma, tamanho e cristalinidade, em escala nano e micrométrica. Uma área específica (marcada por um quadrado pontilhado), que parece ser um cristal único, foi selecionada e investigada utilizando-se SINS. A seleção se baseou no tamanho do cristal (micrômetros), tornando possível o uso prévio de microespectroscopia Raman para caracterizar a estrutura a ser investigada por SINS e facilitar a interpretação dos resultados.

Espectros Raman foram obtidos diretamente da microestrutura (Figura 2) em duas diferentes orientações em relação ao seu eixo de crescimento, enfatizando seu efeito no perfil espectral, uma vez que as intensidades de bandas Raman em cristais dependem da polarização do laser e da orientação do cristal. Os resultados mostram-se compatíveis com os espectros Raman do formiato 1 (Tabela 1) e a baixa relação sinal/ruído (SNR) observada no espectro do cristal (linha de cor vermelha) se deve ao pequeno número de espalhadores analisado pelo laser, considerando a pequena espessura da microestrutura.

Imagem AFM e respectiva imagem IR de banda larga com melhor resolução espacial foram obtidas do cristal selecionado (Figura 3a e Figura 3b, respectivamente). Na imagem IR de banda larga, o contraste entre as estruturas e o substrato de Si é majoritariamente governado por dois fatores: i) variações na permissividade dielétrica da superfície analisada; e ii) a convolução da resposta espectral do detector com bandas de absorção no IR da amostra. O conteúdo orgânico do cristal causa uma diminuição drástica no sinal comparado à resposta do substrato de $\mathrm{Si}$, mostrando uma correlação invertida

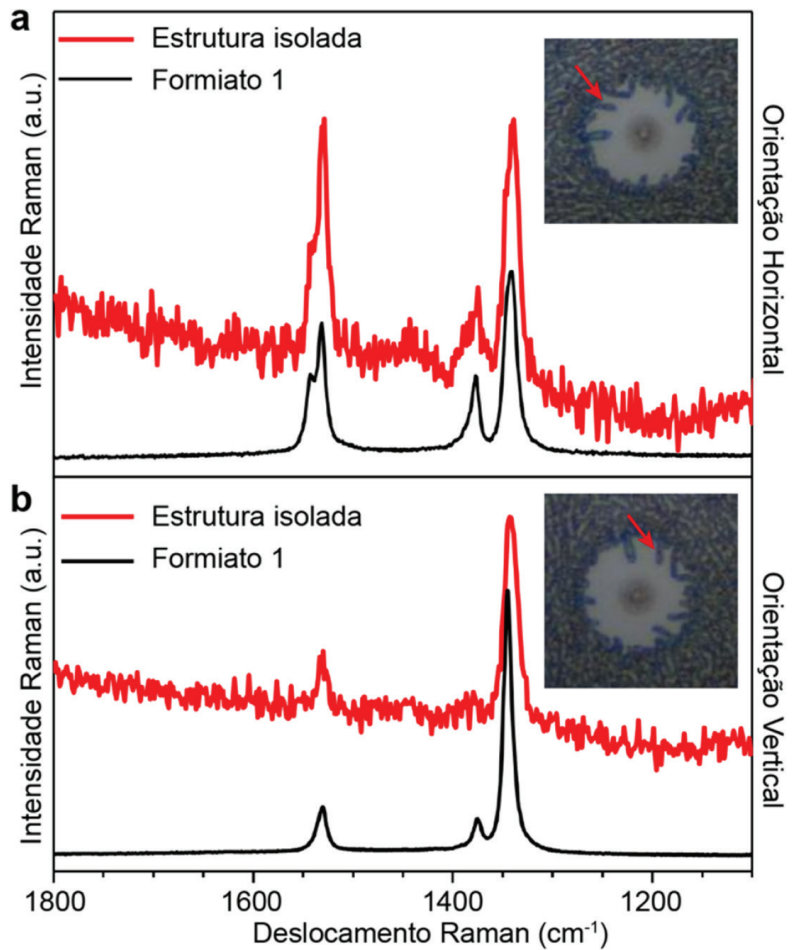

Figura 2. Espectros Raman médios $(632,8 \mathrm{~nm})$ da microestrutura selecionada (linhas de cor vermelha) e do formiato 1 (linhas de cor preta), em duas diferentes orientações do cristal em relação ao seu eixo de crescimento
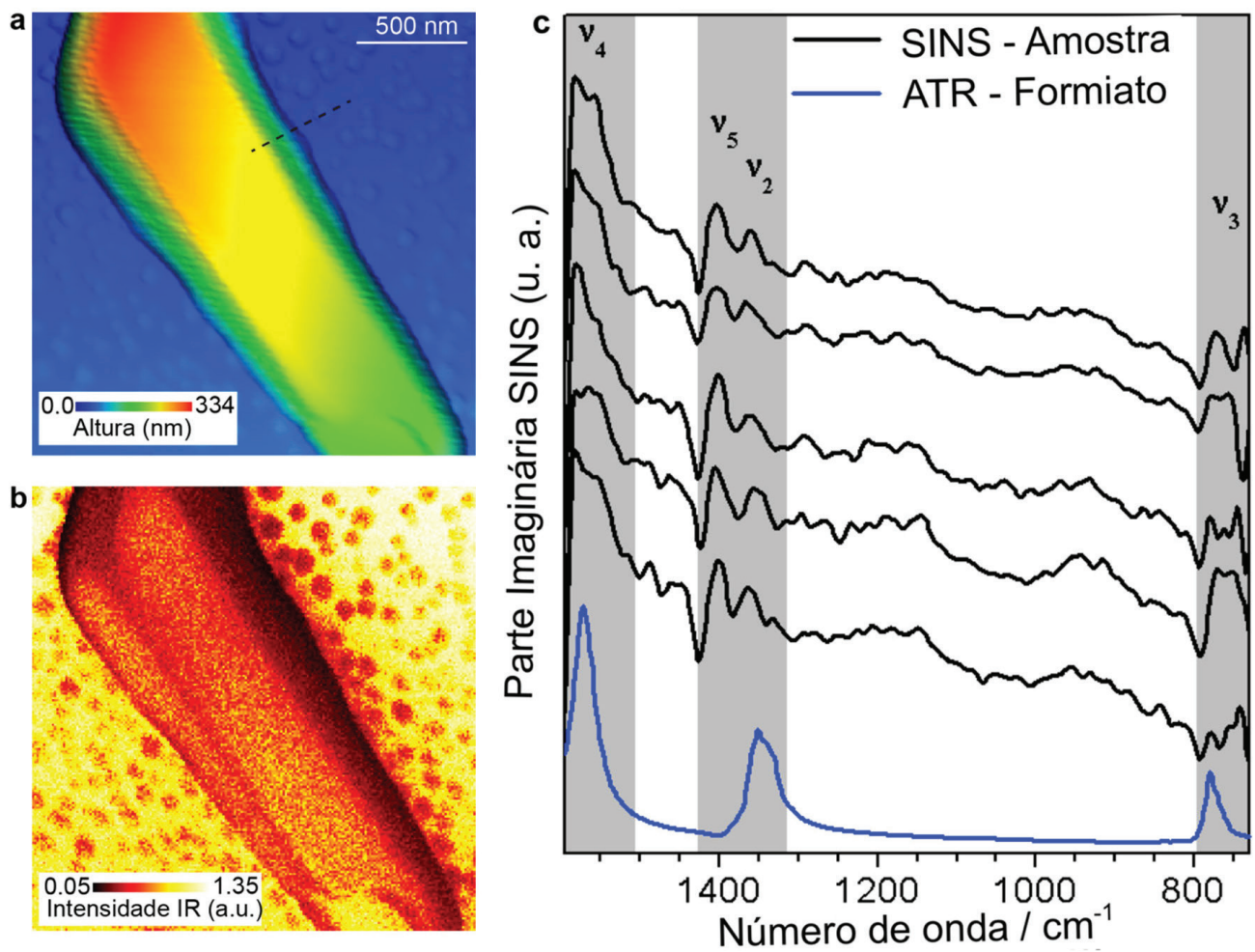

Figura 3. Imagem AFM (a) e respectiva imagem IR de banda larga (b) de uma estrutura isolada produzida após corrosão do filme de Pb (referente ao quadrado branco pontilhado da Figura $1 b$ ). Em (a) mostra-se a região onde conduziu-se investigação por SINS no modo de varredura em linha, representado por um traço pontilhado; (c) mostra os cinco primeiros espectros pontuais de SINS obtidos no modo de varredura em linha (curvas em preto) e o espectro FTIR-ATR de amostra de formiato 1 (curva em azul) 
entre a topografia e os sinais ópticos (quanto maior a altura indicada na imagem AFM, menor a intensidade de IR refletido indicada na imagem de banda larga). Embora as técnicas AFM e micro-espectroscopia Raman indiquem que a microestrutura seja um cristal bem definido e sem impurezas em sua superfície, as medidas do s-SNOM mostram variações do sinal óptico no cristal, sugerindo a presença de nano-heterogeneidades químicas e/ou físicas, ou nano-defeitos.

Para investigar a natureza desta nano-heterogeneidade sugerida por s-SNOM, medidas SINS de uma região selecionada (indicada na Figura 3a) foram realizadas no modo de varredura em linha, com a aquisição de 10 espectros. A Figura $3 \mathrm{c}$ mostra os cinco primeiros espectros obtidos (parte imaginária do sinal de espalhamento transformado por método de Fourier), uma vez que os demais não evidenciaram bandas de absorção na região estudada. O sinal do s-SNOM após a aplicação da transformada de Fourier é uma quantidade complexa que é relacionada à função dielétrica complexa do material, ${ }^{40,41}$ cuja parte imaginária, também representada pela fase do sinal transformado, pode ser correlacionada ao espectro de absorção de campo distante do material. ${ }^{13}$

O perfil dos espectros obtidos assemelha-se ao espectro FTIR-ATR do formiato 1 (Figura 3c), composto este indicado por micro-espectroscopia Raman, apesar do deslocamento de algumas bandas e o aparecimento de outras, o que sugere que mais de um tipo de formiato de $\mathrm{Pb}$ possa estar presente ou que haja mais de um grupo formiato na cela unitária do sólido formado na fina camada de produto de corrosão. A banda em $c a .1583 \mathrm{~cm}^{-1}$, por exemplo, aparece em todos os espectros, bem como a em $c a$. $1557 \mathrm{~cm}^{-1}$. Nota-se, contudo, que a relação de intensidade entre essas bandas não se mantém, sugerindo serem referentes a espécies distintas. Nessa mesma região em um dos espectros nota-se a presença de uma banda em $c a .1566 \mathrm{~cm}^{-1}$, provavelmente referente à um terceira espécie de formiato. Essas bandas em $c a$. 1530 e $1580 \mathrm{~cm}^{-1}$ podem ser atribuídas ao estiramento antissimétrico OCO $\left(\right.$ modo $\left._{4}\right)$ do íon formiato, enquanto bandas em ca. $1350 \mathrm{~cm}^{-1}$ podem ser atribuídas ao estiramento simétrico OCO (modo $v_{2}$ ) e as em $c a .1390 \mathrm{~cm}^{-1}$ à deformação linear antissimétrica OCO $\left(\right.$ modo $v_{5}$ ) do mesmo íon. Bandas na região de $760 \mathrm{~cm}^{-1}$ também podem ser relacionadas à deformação simétrica $\mathrm{OCO}$, atribuída ao modo $v_{3}$ do íon.

É interessante notar que não se observou correlação da variação espectral com a morfologia do cristal, sugerindo que a heterogeneidade está randomicamente distribuída. Vale destacar que as correlações dos espectros SINS com espectros FTIR precisam ser realizadas com o uso de amostras padrão, de modo que mais estudos são necessários para avaliar a aplicabilidade e os limites da técnica para este tipo de sistema.

Conforme esperado, não há bandas de absorção nos espectros obtidos nas áreas de Si exposto, visto que esse material apresenta resposta óptica plana no intervalo espectral de IR considerado. No entanto, a ausência de bandas na região da nanoestrutura vizinha do cristal, que se supunha ser composta do mesmo formiato (ou de formiato análogo), não era esperada. Uma possível explicação para essa ausência é que essa estrutura pode se tratar de um óxido, também com baixa refletividade de IR, mas com bandas de absorção presente em região espectral de baixa frequência, fora da região investigada.

Para melhor visualização da variação espectral na varredura em linha, a Figura 4 mostra o perfil topográfico da linha varrida junto da representação 2D dos espectros versus o passo na linha.

As variações em forma e número de onda dessas bandas sugerem fortemente a heterogeneidade na superfície do cristal, em acordo com os resultados obtidos por s-SNOM. Embora essa heterogeneidade não tenha sido observada nas medidas feitas por micro-espectroscopia Raman, é necessário considerar que a área investigada no Raman é pelo menos cerca de $1 \mu \mathrm{m}^{2}$, ou seja, na mesma ordem de tamanho do cristal. Deste modo, a micro-espectroscopia Raman daria informação sobre componentes/defeitos minoritários somente se eles fossem espalhadores muito mais eficientes do que os componentes presentes em maior quantidade, e aparentemente esse não é o caso. Por outro lado, SINS investiga áreas de $c a$. 0,002 $\mu \mathrm{m}^{2}$, fazendo possível a diferenciação de heterogeneidades bastante localizadas, pertencentes ao cristal ou a espécies que começam a crescer em sua superfície.

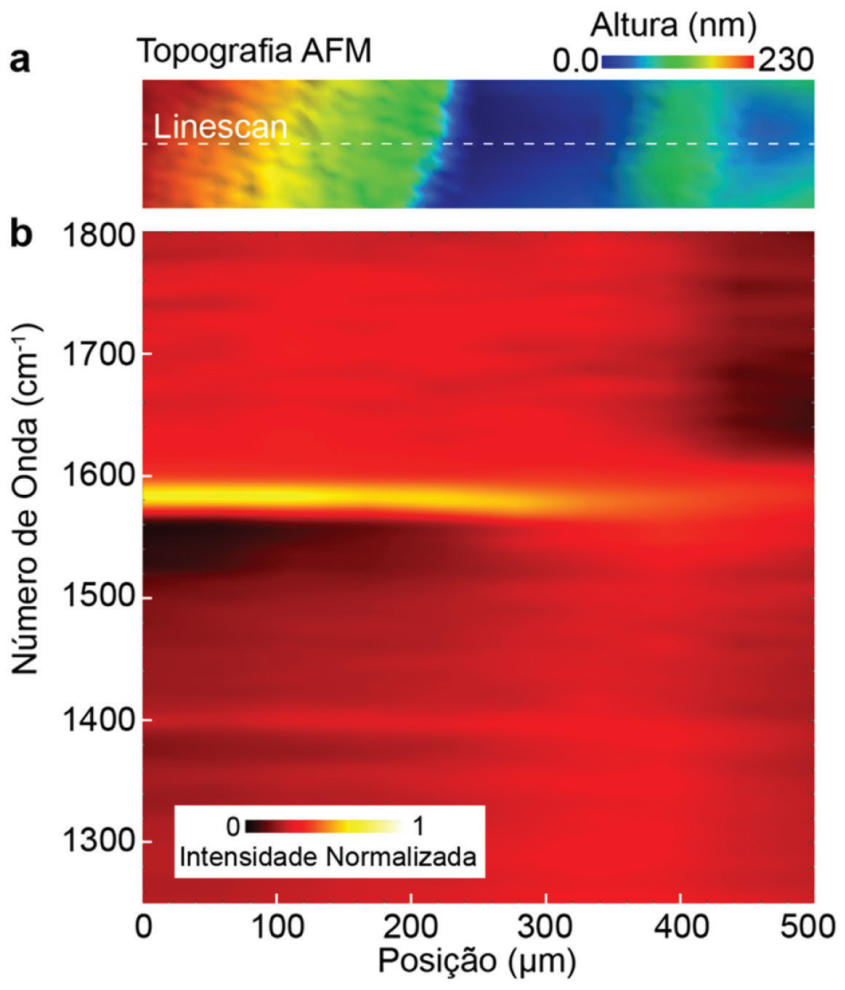

Figura 4. (a) Perfil topográfico da linha varrida da região indicada na Figura 3 a e (b) representação $2 D$ dos espectros obtidos versus o passo na linha

\section{CONCLUSÕES}

Os resultados mostram o potencial de SINS para investigações de corrosão metálica, permitindo nanoespeciação mesmo em estruturas de um único microcristal e complementando investigações feitas por técnicas como a micro-espectroscopia Raman e, potencialmente, TERS. Destaca-se que tais resultados foram obtidos quando o equipamento SINS estava em fase de implantação no LNLS, sendo que atualmente o sistema está completamente implantado e pode ser de muita utilidade numa vasta gama de problemas químicos.

Embora técnicas de caracterização molecular com resolução micrométrica (como micro-espectroscopia Raman) e investigações morfológicas com resolução nanométrica (como AFM) possam indicar homogeneidade na superfície de um cristal, investigações complementares por SINS e s-SNOM podem revelar heterogeneidades, informação muitas vezes essencial para o devido entendimento da reatividade química e do comportamento físico dos materiais.

Apesar de recentemente um estudo ter focado na aplicação de nano-FTIR em corrosão metálica de $\mathrm{Cu}$, analogamente ao aqui apresentado, destaca-se que não foi utilizada radiação síncrotron.$^{42}$ É muito importante ressaltar que a técnica SINS corresponde à combinação de s-SNOM com uma fonte do síncrotron de radiação no infravermelho. As características de alta intensidade, em amplo espectro, e razoável colimação do feixe infravermelho inerentes à radiação síncrotron são pontos essenciais que permitem, à técnica SINS, superar a importante limitação de se sondar baixíssimos volumes de material, 
ca. 2,0 $\times 10^{-4} \mu \mathrm{m}^{3}$, a fim de se obter a alta resolução espacial em torno $25 \mathrm{~nm}$. Essas limitações são, praticamente, intransponíveis a outras fontes de infravermelho de banda comparável à do síncrotron, como fontes térmicas comumente usadas em espetrômetros FTIR convencionais. Fontes térmicas têm intensidade até $10^{3}$ vezes menor ${ }^{23}$ e feixes altamente divergentes em comparação com o síncrotron. De forma complementar, amplificadores e osciladores paramétricos ópticos (OPAs e OPOs, respectivamente) baseados em lasers de pulsos curtos têm sido empregados em s-SNOM, essencialmente para a construção de imagens de banda estreita assim como nanoespectroscopia em faixa limitadas de IR. As bandas de IR de OPAs e OPOs, embora sintonizáveis, são de 3 a 4 vezes mais estreitas que as do síncrontron, o que restringe a faixa de modos vibracionais que se pode investigar em um único experimento. Estes pontos comparativos esclarecem algumas razões da técnica SINS estar, progressivamente, se popularizando e evoluindo.

\section{AGRADECIMENTOS}

Os autores expressam seus agradecimentos à equipe do CNPEMLNLS pelo suporte durante as medidas, aos revisores deste manuscrito pelas contribuições e à FAPESP (2011/13760-8, 2012/13119-3 e 2016/21070-5) pelos financiamentos.

\section{REFERÊNCIAS}

1. Marcus, P.; Mansfeld, F.; Analytical methods in corrosion science and engineering; CRC Press: Boca Raton, 2006.

2. Hosseinpour, S.; Johnson, M.; Materials (Basel). 2017, 10, 413.

3. Martina, I.; Wiesinger, R.; Schreiner, M.; J. Raman Spectrosc. 2013, 44, 770 .

4. Neff, D.; Bellot-Gurlet, L.; Dillmann, P.; Reguer, S.; Legrand, L.; J. Raman Spectrosc. 2006, 37, 1228.

5. Réguer, S.; Neff, D.; Bellot-Gurlet, L.; Dillmann, P.; J. Raman Spectrosc. 2007, 38, 389.

6. Puglieri, T. S.; De Faria, D. L. A.; Cavicchioli, A.; Vib. Spectrosc. 2014, 71.

7. De Faria, D. L. A.; Cavicchioli, A.; Puglieri, T. S.; Vib. Spectrosc. 2010, 54.

8. Sala, O.; Fundamentos da Espectroscopia Raman e no Infravermelho; $2^{\mathrm{a}}$. ed., Editora UNESP: São Paulo, 2008.

9. Wu, C.-Y.; Wolf, W. J.; Levartovsky, Y.; Bechtel, H. A.; Martin, M. C.; Toste, F. D.; Gross, E.; Nature 2017, 541, 511

10. Knoll, B.; Keilmann, F.; Nature 1999, 399, 7.

11. Zenhausern, F.; Martin, Y.; Wickramasinghe, H. K.; Science 1995, 269, 1083.

12. Keilmann, F.; Hillenbrand, R.; Philos. Trans.: Math., Phys. Eng. Sci. 2004, 362, 787.

13. Huth, F.; Govyadinov, A.; Amarie, S.; Nuansing, W.; Keilmann, F.; Hillenbrand, R.; Nano Lett. 2012, 12, 3973.

14. Pollard, B.; Muller, E. A.; Hinrichs, K.; Raschke, M. B.; Nat. Commun. 2014, 5, 3587 .

15. Amarie, S.; Zaslansky, P.; Kajihara, Y.; Griesshaber, E.; Schmahl, W. W.; Keilmann, F.; Beilstein J. Nanotechnol. 2012, 3, 312.

16. Chen, J.; Badioli, M.; Alonso-González, P.; Thongrattanasiri, S.; Huth, F.; Osmond, J.; Spasenović, M.; Centeno, A.; Pesquera, A.; Godignon, P.; Elorza, A. Z.; Camara, N.; García de Abajo, F. J.; Hillenbrand, R.; Koppens, F. H. L.; Nature 2012, 487, 77.
17. Dai, S.; Ma, Q.; Liu, M. K.; Andersen, T.; Fei, Z.; Goldflam, M. D.; Wagner, M.; Watanabe, K.; Taniguchi, T.; Thiemens, M.; Keilmann, F.; Janssen, G. C. A. M.; Zhu, S.-E.; Jarillo-Herrero, P.; Fogler, M. M.; Basov, D. N.; Nat. Nanotechnol. 2015, 10, 682.

18. Patoka, P.; Ulrich, G.; Nguyen, A. E.; Bartels, L.; Dowben, P. A.; Turkowski, V.; Rahman, T. S.; Hermann, P.; Kästner, B.; Ulm, G.; Rühl, E.; Opt. Express 2016, 24, 1154.

19. Bechtel, H. A.; Muller, E. A.; Olmon, R. L.; Martin, M. C.; Raschke, M. B.; Proc. Natl. Acad. Sci. U. S. A. 2014, 111, 7191.

20. Barcelos, I. D.; Cadore, A. R.; Campos, L. C.; Malachias, A.; Watanabe, K.; Taniguchi, T.; Barbosa Maia, F.; Freitas, R. D. O.; Deneke, C.; Maia, F. C. B.; Freitas, R. D. O.; Deneke, C.; Nanoscale 2015, 7, 11620.

21. Johns, R. W.; Bechtel, H. A.; Runnerstrom, E. L.; Agrawal, A.; Lounis, S. D.; Milliron, D. J.; Nat. Commun. 2016, 7, 11583.

22. Pollard, B.; Maia, F. C. B.; Raschke, M. B.; Freitas, R. O.; Nano Lett. 2016, 16, 55.

23. Duncan, W. D.; Williams, G. P.; Hill, B.; National, B.; Appl. Opt. 1983, 22 .

24. Tétreault, J.; Cano, E.; van Bommel, M.; Scott, D.; Dennis, M.; BarthésLabrousse, M.-G.; Minel, L.; Robbiola, L.; Stud. Conserv. 2003, 48, 237.

25. Niklasson, A.; Johansson, L.-G.; Svensson, J.-E.; J. Electrochem. Soc. 2007, 154, C618.

26. Bacci, M.; Cucci, C.; Mencaglia, A. A.; Mignani, A. G.; Sensors 2008, 8,1984

27. Grasseschi, D.; Bahamon, D. A.; Maia, F. C. B.; Neto, A. H. C.; Freitas, R. O.; de Matos, C. J. S.; 2 D Mater. 2017, 4.

28. Pereira, L.; Flores-Borges, D. N. A.; Bittencourt, P. R. L.; Mayer, J. L. S.; Kiyota, E.; Araújo, P.; Jansen, S.; Freitas, R. O.; Oliveira, R. S.; Mazzafera, P.; Plant Physiol. 2018, 177, 1629.

29. Dery, S.; Kim, S.; Haddad, D.; Cossaro, A.; Verdini, A.; Floreano, L.; Toste, F. D.; Gross, E.; Chem. Sci. 2018, 9, 6523.

30. Wiens, R.; Findlay, C. R.; Baldwin, S. G.; Kreplak, L.; Lee, J. M.; Veres, S. P.; Gough, K. M.; Faraday Discuss. 2016, 187, 555

31. Cotte, M.; Dumas, P.; Taniguchi, Y.; Checroun, E.; Walter, P.; Susini, J.; Comptes Rendus Phys. 2009, 10, 590.

32. Cotte, M.; Radepont, M.; Pouyet, E.; Salome, M.; Susini, J. In Trends of Synchrotron Radiation Applications in Cultural Heritage, Forensics and Materials Science; International Atomic Energy Agency, 2016.

33. Hermann, P.; Hoehl, A.; Patoka, P.; Huth, F.; Rühl, E.; Ulm, G.; Opt. Express 2013, 21, 2913.

34. Freitas, R. O.; Deneke, C.; Maia, F. C. B.; Medeiros, H. G.; Moreno, T.; Dumas, P.; Petroff, Y.; Westfahl, H.; Opt. Express 2018, 26, 11238.

35. Puglieri, T. S.; Tese de Doutorado, Universidade de São Paulo, Brasil, 2015.

36. Moreno, T.; Westfahl, H.; Freitas, R. D. O.; Petroff, Y.; Dumas, P.; J. Phys. Conf. Ser. 2013, 425, 142003.

37. Freitas, R. O.; Maia, F. C. B.; Deneke, C.; Moreno, T.; Dumas, P.; Westfahl, H.; Petroff, Y.; Synchrotron Radiat. News 2017, 30, 24.

38. Huth, F.; Schnell, M.; Wittborn, J.; Ocelic, N.; Hillenbrand, R.; Nat. Mater. 2011, 10, 352.

39. Ayyub, P.; Chandra, R.; Taneja, P.; Sharma, A. K.; Pinto, R.; Appl. Phys. A: Mater. Sci. Process. 2001, 73, 67.

40. Govyadinov, A. A.; Amenabar, I.; Huth, F.; Carney, P. S.; Hillenbrand, R.; J. Phys. Chem. Lett. 2013, 4, 1526.

41. Xu, X. G.; Rang, M.; Craig, I. M.; Raschke, M. B.; J. Phys. Chem. Lett. 2012, 3, 1836.

42. Johnson, C. M.; Böhmler, M.; Corros. Sci. 2016, 60.

FAPESP contribuiu para custear a publicação deste artigo. 